\title{
Enabling Component-Based Design for Embedded Real-Time Software
}

\author{
Jimmie Wiklander, Jens Eliasson, Andrey Kruglyak, Per Lindgren, Johan Nordlander \\ Dept. of Computer Science and Electrical Engineering \\ Luleå University of Technology \\ Luleå, Sweden \\ Email: \{jimmie.wiklander, jens.eliasson, andrey.kruglyak, per.lindgren, johan.nordlander\}@ltu.se
}

\begin{abstract}
The increasing complexity of embedded software calls for a new, more efficient design approach. A natural choice is to use well-established component-based design; however, its adoption to design of embedded software has been slow and riddled with difficulties. It can be argued that these problems are due to the following peculiarities of embedded systems. Firstly, the tight integration between hardware and software, typical for embedded systems, makes it virtually impossible to model and implement software separately from hardware. Secondly, it is difficult to express timing requirements, an intrinsic part of functionality of many embedded systems, in dataflow abstractions traditionally used in component-based design.

We propose to overcome these difficulties by introducing a uniform, consistent modeling of both hardware and software and by integrating timing requirements into the model. We present a modeling framework based on the notions of reactive objects and time-constrained reactions, which enables component-based design of embedded real-time systems. Within this framework, functionality of both hardware and software components is defined in terms of reactions to discrete external events, and timing requirements are specified for each reaction relative to the event that triggered it. We also present a detailed software design methodology for embedded real-time systems based on our modeling framework.
\end{abstract}

Index Terms-component-based design, embedded realtime systems, embedded software, reactive objects, timeconstrained reactions

\section{INTRODUCTION}

In recent years, the complexity of embedded systems has been steadily increasing, and the number and complexity of functions performed by embedded software has also grown. This calls for introduction of new, more efficient design methods ${ }^{1}$. An attractive approach is component-based design, which facilitates component reuse, separate development of components, and improves overall maintainability and robustness of the system.

However, adoption of this approach to embedded software development has been significantly slower than to software development in general. It can be argued that the problem lies in the fact that embedded systems manifest a tight integration between functionality implemented in software and functionality of hardware parts. In many embedded systems, hardware components cannot be viewed

\footnotetext{
${ }^{1} \mathrm{~A}$ good overview of existing design practices and research trends in embedded system design is given in [1] and [2].
}

as part of the environment external to the software system since the software has to be developed "around" the available hardware resources, relying on their timing and other properties. This requires a uniform, consistent modeling of both hardware and software. The situation is further complicated by the fact that embedded systems, unlike most general-purpose computing systems, often perform computations subject to various constraints, such as processor speed, amount of memory, power consumption, and reaction time. The timing requirements are often of special importance, especially for safety-critical systems. In fact, the majority of embedded systems can be viewed as real-time systems, i.e. systems in which correctness of system behavior (for hard real-time systems) or quality of service (for soft real-time systems) relies on the time when results are delivered to the environment as well as on the computed values as such.

We conclude that it is necessary to modify the traditional component-based approach to software development so that (a) a tight integration between software and hardware is taken into account, and (b) timing requirements can be clearly defined at both system and component level and used to guide implementation.

In this article we present a modeling framework that allows to uniformly model both hardware and software and to incorporate timing requirements into the model (Section II). We also present a step-by-step methodology for embedded software design based on our modeling framework (Section III) and demonstrate it in the design of a small embedded system, a personal alarm device (Section V), implemented in the programming language Timber (Section IV). A short overview of related work is given in Section VI.

\section{MOdeling FramewORK}

Component-based design relies on the existence of consistent and coherent models of individual components that can be composed to model the whole system. We propose a modeling paradigm based on a combination of event-based, reactive, concurrent, and object-oriented programming models that provides a natural framework for specifying the behavior of hardware, software, and mixed hardware/software components of an embedded system. 


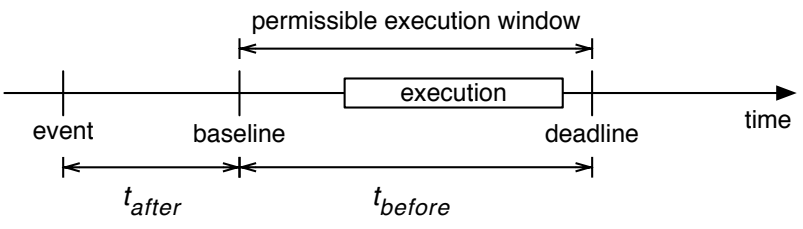

Figure 1. Permissible execution window for a reaction to an event.

Event-based modeling implies that interaction between the system and its environment, as well as between components of the system is conducted by means of discrete events occurring at specific times. The reactive approach allows us to specify functionality in terms of reactions to such events, and since both input and output events are discrete, it is possible to impose time constraints on these reactions, effectively integrating timing requirements into functional specification [3]. The simplest way to specify such constraints is by defining the earliest and the latest reaction time (baseline and deadline) relative to the time of the input event triggering the reaction. We will call the time window between the reaction baseline and its deadline a permissible execution window for this reaction (Fig. 1) and denote it as after $t_{\text {after }}$ before $t_{\text {before }}$ doSmth, where $t_{\text {after }}$ is the period of time between the event and the baseline, $t_{\text {before }}$ is the period of time between the baseline and the deadline, and doSmth is the invoked method.

Concurrency is inherent in hardware and is unavoidable in more complex software systems that have to perform multiple tasks (react to multiple events) at the same time. It is important to reflect this concurrency in the model of an embedded system. This gives rise to the problems of synchronization and state protection. We address these issues by modeling and implementing components using reactive objects ${ }^{2}$ [4]; we define that all mutable state variables have to be encapsulated within an object and only accessible via its methods. Reactive objects can be

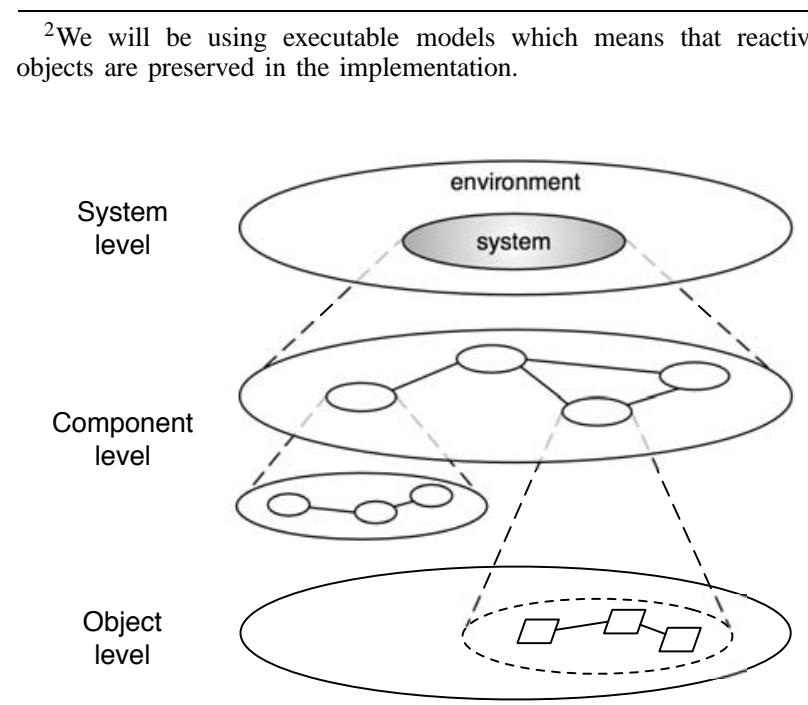

Figure 2. Three abstraction levels of modeling: system level, component level (including multiple sublevels to accommodate a component hierarchy), and object level. A system is realized in terms of components, and each component is realized in terms of objects.

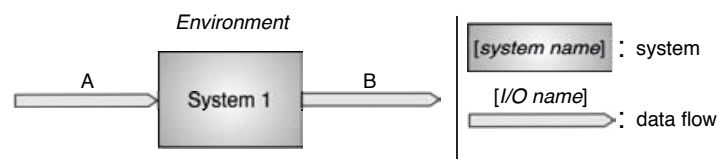

Figure 3. Data flow model of system interaction with its environment.

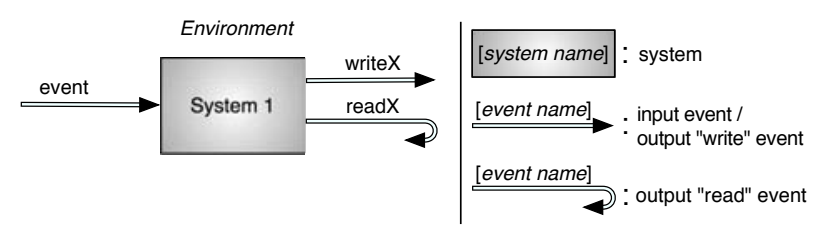

Figure 4. Event-based system-level model.

used as units of concurrency by specifying that no two methods of the same object can execute concurrently while any two methods of different objects can.

Modeling complex systems requires using multiple levels of abstraction. We will distinguish the following abstraction levels: system level, component level (which can include multiple sublevels to accommodate a hierarchy of components), and object level, as depicted in Fig. 2. In our model, we will not try to include all information at each level; instead, the relationship between the layers is one of a gradual refinement of the model where each next level contains more details.

\section{A. System-Level Model}

At system level, the system is viewed as a black box, and the focus is on defining the boundary between the system and its environment. Since embedded systems typically manifest a tight integration between software and hardware, the system model should include both software and hardware, even if the hardware is given and is not developed as part of the design process. From the modeling perspective, existing hardware parts can be considered either as a part of the system or as a part of its environment. In this case, the system boundary should be defined so that it is easy to specify system functionality in terms of reactions to input events as described below.

In component-based design, the system's interaction with the environment is typically described in dataflow terms as input from the environment and output from the system (Fig. 3). However, to be able to define timing properties of the system, input and output should be expressed as discrete events occurring at specific times, resulting in a reactive event-based model. Then system functionality can be defined as reactions to input events and timing requirements can easily be described as constraints on these reactions. Output events constitute part of a system reaction to an input event and can be divided into asynchronous ("write") and synchronous ("read") events (Fig. 4). Note that if some parameter in the environment is sampled by the system, this can be reflected as an input in the dataflow model but as a "read" output event in the event-based model. 


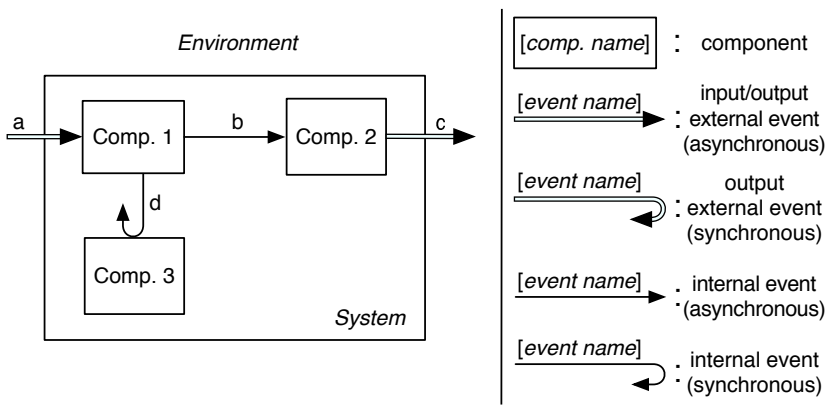

Figure 5. Event-based component-level model.

\section{B. Component-Level Model}

At component level, we use components to model the system. A component is defined as an encapsulation of a part of system state and/or hardware resources, with a clearly defined interface and functionality. Importantly, state variables and hardware resources must belong to only one component and cannot be shared by two or more components (the question of allocating CPU resources, i.e. processing time, to components will be addressed later). This definition allows for hardware, software, and mixed hardware/software components.

Each component can be specified independently of the rest of the system in terms of time-constrained reactions to input events. Input events can either be external events originating outside the system, or internal events originating in another component. Both input and output events can be asynchronous ("write" events, one-way interaction) or synchronous ("read" events, synchronization events, etc.). Note, however, that external input events are always asynchronous (Fig. 5). Unlike reactions to asynchronous events, reactions to synchronous events cannot have a permissible execution window of their own, as they have to complete before the deadline for the component that posted the synchronous event and awaits a response.

Components can be organized hierarchically, when a component is partitioned into subcomponents. Partitioning is governed by considerations such as composability, reusability, ease of understanding, etc. as will be described later in this article.

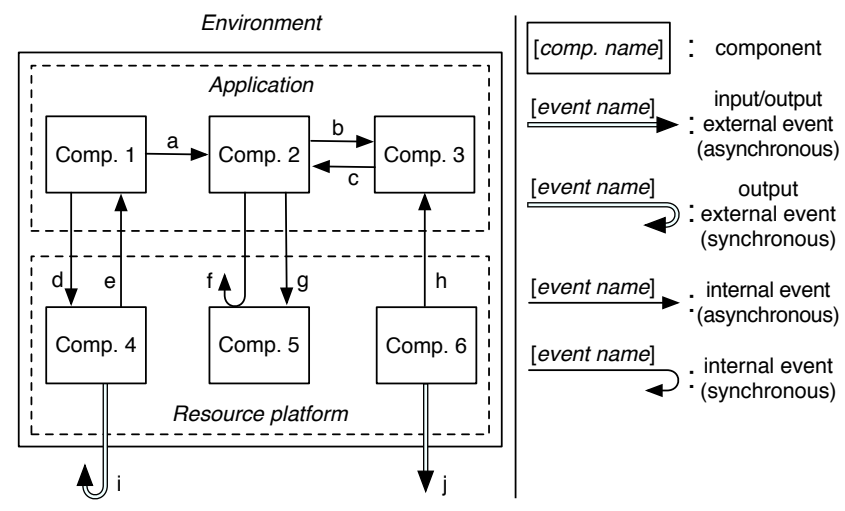

Figure 6. Partitioning of a system into a resource platform and a software application.

\section{Resource Platform}

A useful abstraction that can be built upon system partitioning into components is the notion of a resource platform. The intuition behind it is that a number of components taken together can present a certain basic functionality with a clearly defined interface that can be utilized by a whole range of applications (Fig. 6). A resource platform typically includes all hardware components of the system, since they are the most difficult to change and may often have somewhat limited composability, but it can equally well include mixed hardware/software components or software-only components that are used as resources by the applications. This gives us a clear separation of the system into a resource platform and an application (embedded systems typically have only one application, but it is possible to consider several applications sharing the same platform at runtime).

Note that since the separation into a platform and an application is performed at a relatively high level of abstraction, a platform may have multiple instances, differing in the choice of specific hardware and/or specific implementation of software. This approach allows for a fast and efficient development of a number of applications for a certain platform while leaving enough flexibility in platform implementation to perform optimizations in device size, cost, power consumption, and performance.

\section{Object-Level Model}

At the lowest level, each component is modeled using reactive objects. A reactive object is a model that can have one or several hardware and/or software instances; however, it cannot be instantiated as a mixed hardware/software entity. The choice of implementation is

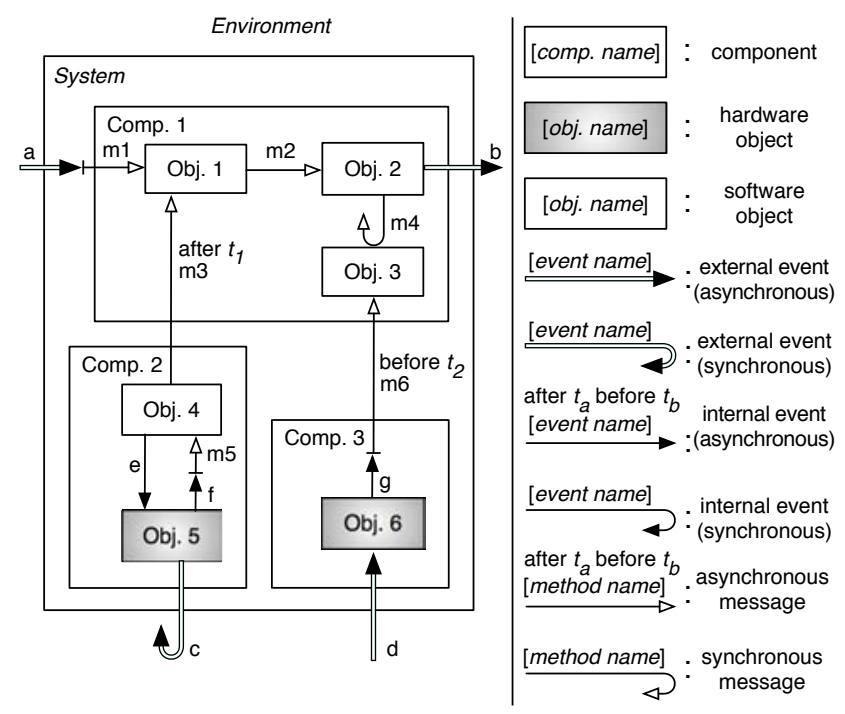

Figure 7. Event-based object-level model. For each reaction, a permissible execution window can be specified using the after $t_{a}$ before $t_{b}$ notation; absence of such notation indicates inheritance of timing constraints. Input events to software objects originating in other software objects are marked as messages; input events to software objects originating outside the system or in hardware objects are translated into messages. 
made at this level, so an object-level model clearly specifies which objects should be implemented in hardware and which in software (Fig. 7).

Both software and hardware objects react to external and internal input events and for each reaction a permissible execution window can be specified relative to the time of the event (Fig. 1). External input events originate in the environment, and each type of event triggers a method of a specific object. Internal input events originate within the system; in the case when such events are both produced and consumed by software objects, they can be viewed and implemented as messages. Even events originating outside the system or in hardware objects can be translated into messages if they are consumed by a software object.

As any events, messages can be either synchronous or asynchronous. In the latter case, a software object can also post a message to itself. Asynchronous messages can be delayed by a certain amount of time defined relative to the baseline of the object sending the message. This also allows to encode a periodic behavior by letting an object post a delayed asynchronous message to itself. Synchronous messages return a value, and the execution of the sender object is blocked until then; that is why reactions to synchronous messages cannot be delayed and always inherit the permissible execution window of the sender.

A software object encapsulates its state and provides methods to operate on it; a reactive software object cannot block during method execution waiting for input. In our model, state protection is absolute - all mutable variables have to be state variables in some object, and no access to state variables is allowed except via methods of the object. Besides, no two methods of the same object are allowed to execute concurrently but methods of two different objects can, resulting in an object-level concurrency model.

\section{Software Design Methodology}

Here we present a methodology for embedded software design based on the modeling framework described in the previous section. This framework allows us to model both software and hardware parts of an embedded system, and a complete model of the system is essential for designing embedded software and verification of the system as a whole.

The different stages of the design process are presented in Fig. 8. The input to the design process is the product specification which originates from the client commissioning the system and which we assume to be static during the development of the system. This specification is usually written in a natural language, is often incomplete and imprecise. Hence the first step is drawing up a complete specification with a clear division into functional and non-functional parts. In our case, functional specification is integrated with timing requirements and is used throughout system modeling and implementation. Non-functional specification lists the remaining system properties and constraints, such as system size and power

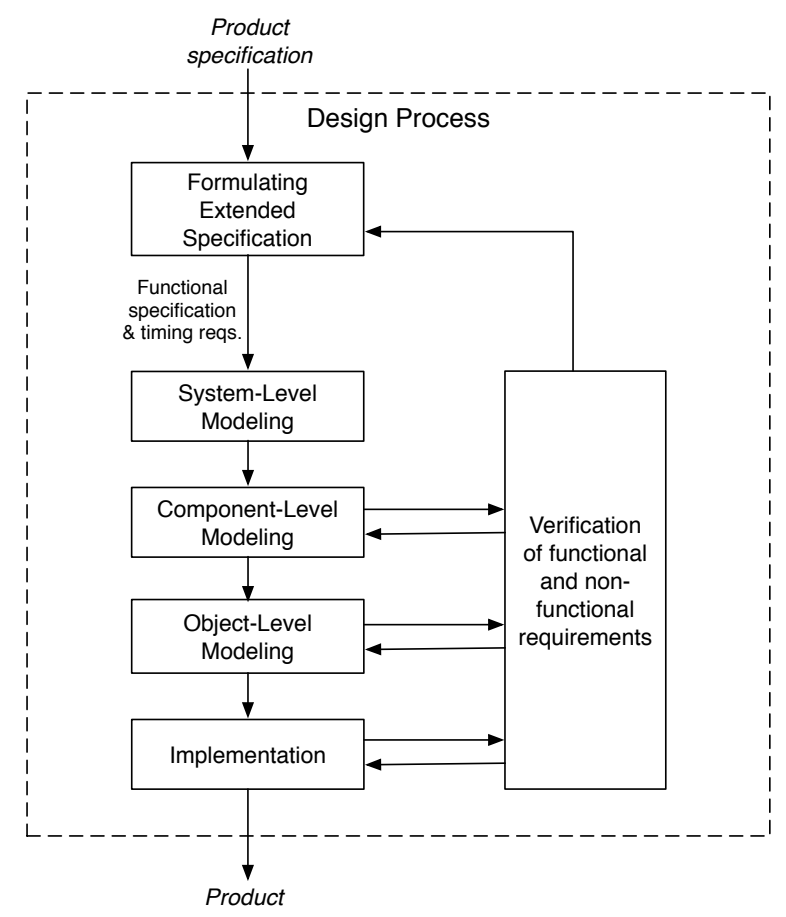

Figure 8 . Stages in the design process: from a specification to a ready product.

consumption, and is primarily used during verification. It can also be used to guide selection of ready-made components, especially those including hardware.

The second step is formulating a system-level model where system interface to its environment is defined in terms of external input events triggering time-constrained system reactions and system output events which are part of such reactions. In the third step, this model is elaborated by identifying system components and interfaces between them. Such components are key to facilitating software re-use and maintenance, as well as system verification. In the fourth step, the components are realized using reactive objects, and a decision is made on which reactive objects should be implemented in software and which represent models of (existing) hardware parts. At every step, the model of each component is matched against a repository of previously developed components (either software or hardware), which should contain reactive models of components alongside their implementation.

The fifth step is implementation of software objects in some programming language. The sixth and final step is system verification, which can be done by simulation of the model, by testing of the implementation, or by formal methods. Both functional and non-functional requirements can be verified, and a failed verification forces a return to an earlier development step, making the development process iterative. Verification of new components should also be performed at earlier stages of development to verify certain properties at object and component levels. At the final step, verification of component integration and of the system as a whole is conducted. Note that verification of system schedulability 
on a particular hardware platform is separate from verification of the model itself.

\section{(1) Defining Extended System Specification}

The extended specification has to be complete, verifiable, and contain a clear separation into functional and non-functional specification; it is obtained by refining the original product specification. Our approach requires that the functional specification should be expressed in terms of time-constrained reactions to external events representing input to the system or changes in the environment. Thus timing requirements are integrated into functional specification. It has to be noted that not all systems let their functionality to be naturally defined in terms of timeconstrained reactions, which should be seen as a limitation of applicability of our approach. However, it is our belief that the majority of embedded systems can be specified in such a way.

A prominent example is a system with a timecontinuous input; a system which as such does not conform to the notions of events and reactions. However, on closer inspection, any discrete realization of such a system would indeed require a sampling strategy with its corresponding timing requirements.

In other cases timing requirements can be implicit, defined by the rate of incoming events and the necessity to keep up with them. A typical example is routing of packets in a network; while the maximum forwarding delay may be omitted from the specification, it can be derived from a packet buffer length together with the allowed drop rate for a given traffic profile.

\section{(2) Formulation of System-Level Model}

In this step a system-level model should be formulated from the functional and timing specification by determining the system's boundary with its environment and its interface. To achieve a clear-cut separation between the system and its environment, the system should be defined to encompass all the functionality that we have to develop, and it should be taken to include the hardware that the developed software will execute on. Such hardware should be notionally included in the system even if it is given and cannot be changed during the development process. Note that the environment includes both natural phenomena the system will interact with and the infrastructure that is being developed or has been developed separately. Thus all "external" services used by the system, especially those shared between the system under development and other systems, are considered to be part of the environment rather than the system proper.

\section{(3a) Partitioning into Components}

Although component-based design has been studied for several decades, partitioning of a system into components (as well as partitioning of a component into subcomponents), remains more of an art than an exact science.
However, it is possible to identify the main guiding principles.

Each component should have a clearly defined role in the system, and a one-to-one mapping between components and system functions is always preferable. This means that any two independent tasks, triggered by independent external events and resulting in independent outputs, should be realized by two separate components. The same is true for the case when a system should perform two activities in parallel, with little or no state sharing and/or interaction between them.

A special type of components (often associated with hardware or mixed hardware/software components) are resources which can be used by one or several activities and which usually enforce some kind of exclusion or sharing protocol to guarantee consistency of system output and/or its internal state. Several resources are often bundled together in one component when they are used jointly to perform one task or cannot operate in parallel.

Apart from these main principles, a number of other considerations can affect the design of a particular system, such as:

- composability - to facilitate system composition from newly-designed or ready-made components, it is important for each component to have a clear purpose (role in the system) and a clearly defined interface. It is also advantageous to have as few interdependencies between components as possible.

- reusability - functionality common to a class of (possible) applications can be effectively assigned to a separate component, facilitating component re-use.

- robustness - to make better use of ready-made components, and to enhance system verification while shortening the development time, it is important that each component is designed with regard to future verification (testing, simulation, and possibly formal verification) at component level as well as at system level. Robustness can also be improved if components are used as fault-containment regions, which requires detectability of errors at component boundaries.

- ease of understanding - an extremely important consideration that is often overlooked is that partitioning into components should enhance the ability of the original developer(s) of the system as well as those who may work with it in the future to clearly understand the functions and structure of the system. This calls for the components to be small enough to be easily comprehendable, but at the same time large enough to keep the structure of the higher-level component simple. Experience shows that following this principle leads to fewer mistakes (and hence shorter development times and increased robustness) and facilitates re-use and maintenance.

An important issue of component-based design is what kind of interactions are allowed between components. It is advantageous to make components as independent of each other as possible since it simplifies component 
specification, enhances composability, and facilitates verification of individual components. We therefore strongly discourage synchronous communication across component boundaries. Synchronous communication between components should normally be used for predictably quick interaction, such as reading a value (as opposed to waiting for a value to be computed), or performing a hardware operation that takes a known time to complete under certain operational conditions.

Once defined and implemented, components can be stored in some repository for future use. It is important to preserve not only the actual implementation, but also a model of the component (see section $3 b$ ) alongside its testing and verification results. It may also be useful to preserve a testing suit for a component so that the tests can be re-run in a new setting. If the implementation of a component is protected as intellectual property and will not be accessible for system verification, the timing properties of the component also have to be stored in the repository. These would have to include execution time and maximum blocking time (per hardware resource) for each reaction defined in the interface of the component.

\section{(3b) Search for Ready-Made Components}

In this step, models of defined components are matched against models of earlier developed components from the repository. Comparison between the models requires that they are of the same kind. In our case, it means that a component model should have its functionality expressed in terms of time-constrained reactions to events external to the component. Identity of modeling principles should lead to a straightforward integration of a matched component into the system model.

There might be components in the repository that do not match the specification, but can be either adapted by introducing an intermediate layer, or can be modified to fit the specification. The downside of component modification is that it may require substantial work on reimplementing the component as well as invalidate the testing and verification results.

\section{(3c) Hierarchical Refinement of Component Structure}

One of the strengths of component-based design is the possibility of hierarchical refinement of component structure. Partitioning of a component into subcomponents closely mirrors partitioning of a system into components as described above; the same principles and guidelines apply. Since one and the same component can (at least theoretically) be used in different systems, partitioning into subcomponents should be performed independently for each component and should not be influenced by a wider context in which the component is used. However, it is possible that identical subcomponents are identified as parts of different components, and those can be viewed as separate instances of the same component class.

If any new subcomponents have been identified in this step, a return to search for matching components in a repository is warranted. The process is repeated until no further refinement of component structure can be justified.

\section{(4) Realization Using Reactive Objects}

The last step in the modeling process is component realization using concurrent reactive objects. This step involves partitioning of the component into reactive objects and identifying hardware and software parts. Similarly to partitioning into subcomponents, it is performed on each component independently of its context. Note that at this level hardware parts are modeled as reactive objects, which allows for a certain flexibility when several hardware parts are modeled using the same object model if they only differ in, for example, power consumption.

For each component, it is necessary to identify: hardware resources; object state in terms of state variables; and object functionality in terms of methods. Partitioning of a component into objects is governed by slightly different principles than partitioning of a system into components. These principles can be obtained by adaptation of wellknown object-orientation strategies to the concept of concurrent reactive objects. The following has to be taken into consideration:

Each object encapsulates its state that can only be accessed by methods of the same object. At the same time, the objects are units of concurrency, meaning that any two methods of the same object cannot be executed concurrently but any two methods of two different objects can. A notable exception is the case when an object posts a synchronous message to another object; then the caller remains blocked until the invoked method returns.

The guiding principles of partitioning into objects aim to maximize schedulability of the system while maintaining state consistency. Component state, seen as a collection of state variables, should be partitioned and assigned to objects in such a way that

- state duplication (when the same state is duplicated as state variables in two or more objects, leading to synchronization problems) is avoided;

- state variables routinely modified together are encapsulated in one object;

- otherwise, state is maximally distributed between different objects to allow for a better schedulability of the system.

Functionality should be assigned to methods, and methods to objects in such a way that

- methods using the same state variables are assigned to the same object;

- methods using different parts of component state are assigned to different objects together with corresponding state variables, in order to maximize schedulability of the system; an exception to this rule is the case when consistency between several state variables has to be guaranteed;

- a special attention is paid to the consequences of mutual exclusion between methods of the same object, when an object remains blocked and cannot execute 
any other method while an earlier invoked method is executing. For example, in some cases a single reaction should be split into two methods, one calling the other asynchronously, thus creating a window of opportunity for a reaction with a shorter deadline to execute on the same object in between the two methods.

The issue of software interaction with hardware parts is of utmost importance and has to be considered separately. This interaction is often governed by complicated protocols that are not relevant to the application at large. Hence it is a good idea to have a single software object controlling access to specific hardware. Apart from providing a useful abstraction of the software-hardware interface, such objects can be used to explicitly control sharing of the hardware resource by enforcing arbitration or queuing if so required.

\section{(5) Implementation}

The next step is the implementation process in which the system model is instantiated. The hardware platform is built using identified hardware parts (COTS components, SoC blocks, etc.), and software reactive objects are implemented in some programming language. In the case when some of the software components are re-used from the repository, the issue of code integration has to be addressed. The complexity of code integration will depend on the language used in the implementation of the re-used components.

An example of a programming language, Timber, fully supporting the described modeling framework and thus suitable for use together with the present software design methodology, will be described in section IV.

\section{(6) Verification}

The final step in embedded system design is verification (see Fig. 8). We will distinguish between verification of the model and verification of the implementation; both should be conducted at component as well as system level.

Verification of the model is done against system specification and specification of individual components. This includes verification of component composition at system level and verification of functional specification (including timing requirements), which can be performed using simulation or with formal methods (see, for example, the work on UPPAAL [5]-[8]). Importantly, verification of the model is independent of its feasibility, i.e. whether or not it can be implemented in a specific programming language and on a specific hardware platform in such a way that the functional and timing requirements are met.

Verification of the implementation should also be conducted at both component and system level and, unlike verification of the model, it involves verification of both functional and extra-functional requirements. At component level, it is only necessary to verify that the implementation corresponds to the model. At system level, both component integration and system feasibility have to be verified. System feasibility refers to the ability of a specific implementation (software and hardware) to meet the functional and timing specifications of the model under extra-functional constraints such as energy consumption; an important part of feasibility verification is schedulability analysis (see [9]). Note that schedulability analysis requires a full knowledge of the system implementation. In the case when the implementation of a particular component is not available for analysis, at least the list of resources used by each reaction of the component should be known together with the execution time and maximum blocking time for each resource. Schedulability analysis should be the preferred way of system verification since it allows to prove system correctness for all inputs and in all situations, as do other formal methods. However, verification of system implementation can also be conducted using simulation and testing.

Let us separately consider verification of a resource platform. A clear division into a platform and an application allows to verify them separately, so that an already verified platform with known properties can be used for development of other applications. It should be noted, however, that system-level verification such as schedulability analysis has to be performed on the system as a whole, including both the application and the resource platform, even if the platform has previously been verified.

\section{AN IMPLEMENTATION APPROACH: THE TIMBER LANGUAGE}

The presented model is sufficiently general to allow a variety of possible implementations. For example, since we can model a complete system including both hardware and software parts, the border between hardware and software can be adjusted even after the model has been completed. Hardware components together with hardware parts of mixed (hardware/software) components can be realized by e.g. selecting existing COTS hardware parts and integrating them into a single hardware platform, whereas software components together with software parts of mixed components have to be implemented in some programming language, typically combined with a minimal operating system or a kernel that will provide scheduling, I/O, etc.

While it is fully possible to implement the model described above in, for example, $\mathrm{C} / \mathrm{C}++$ or Esterel, the translation itself would be far from trivial. The problem is to preserve the properties of individual components and of the system as a whole, to maintain composability of defined components, and to be able to verify that functionality and timing of the resulting code reflect those of the model. Using mainstream programming languages often results in a gap opening up between the model and its implementation. For example, reaction deadlines may have to be translated into thread priorities and as a result, the system's behavior would depend on other tasks and the scheduling policy; hence the correspondence between 
the model and its implementation becomes very difficult to verify.

Another possible implementation approach is to use the recently developed modeling and programming language Timber, which targets real-time systems ([10]-[12]). Timber is a high-level programming language that uses the same primitives as the proposed model, including reactive objects and time-contrained reactions. Hence translation of a model into Timber code is straightforward and preserves system structure and timing specification, closing the gap between the model and its implementation. Timber code can be compiled into a subset of $\mathrm{C}$ and executed on any target platform in combination with a Timber kernel ${ }^{3}$, which uses permissible execution windows preserved in the code for deadline-based scheduling.

Timber is both a high-level programming language for real-time systems and a formalism that can be used to verify a system's functional behavior, timing properties (complying with deadlines), liveness (absence of deadlocks), and termination of computations. Let us briefly describe the relevant properties of the language:

- inherent support for reactivity: the system functionality is expressed in terms of reactions to external events, with reaction defined as a combination of internal state updates and/or system outputs. Each reaction can be comprised by a chain of reactions executed by different objects, some of them executed concurrently. Execution of a system reaction must be non-blocking, i.e. it cannot block waiting for an external input.

- time-constrained reactions: each reaction has a baseline (the earliest time when execution can start) and a deadline (the latest time by which execution must have finished); it is possible to schedule a reaction to start at some point of time in the future by setting its baseline relative to the baseline of the reaction being executed. The timing requirements are preserved in the application code at run-time and can be used to guide scheduling.

- object-orientation: while constants (including global functions) can be defined at the top level, mutable variables are only allowed within objects as state variables. State encapsulation and protection are achieved by limiting access to these variables to the methods of the object, and state consistency is easily guaranteed by always enforcing mutual exclusion between the methods of the same object.

- object-level concurrency: Timber is a highly concurrent language with concurrency achieved by allowing methods of any two different objects to be executed in parallel.

- message passing between objects: Timber objects communicate by passing messages, synchronous (when the sender remains locked and waits for the

\footnotetext{
${ }^{3}$ A prototype version of a Timber kernel has so far been implemented for a generic POSIX environment and for an ARM platform, but thanks to its minimalistic nature it can be ported to other platforms relatively easily.
}

message to return), and asynchronous (when the sender posts a message to another object or to itself, possibly with a postponed baseline, and continues execution). Asynchronous messages lead to concurrent execution of reactions.

\section{AN EXAMPLE SYSTEM: \\ A Personal Alarm Device}

The software design methodology described above has been tested in the development of a personal alarm device, used here to demonstrate different stages in the design process. Some details have been omitted for presentation purposes. The following functional specification of the device was given in the beginning of the design process:

The personal alarm device is a battery-driven system worn by a person on his or her body, for example, by an elderly person at a care facility. The device is capable of detecting the person's fall by analyzing acceleration. Once a fall has been detected, a fall alarm is sent wirelessly to an external receiver. The analysis requires that acceleration is sampled periodically every $t_{\text {period }}$ milliseconds. The device also includes an assistance call button that can trigger a separate kind of alarm sent in the same manner. An alarm must be sent within $t_{\text {alarm }}$ milliseconds after a fall has been detected or after the button has been pressed.

\section{(1) Defining Extended System Specification}

An extended system specification should include both functional and non-functional requirements. The functional requirements have to be expressed in terms of time-constrained reactions. Two such reactions can be identified by analyzing the original specification.

The first reaction is sending an assistance alarm when the push button has been pressed. There is a timing requirement that the alarm is to be sent within $t_{\text {alarm }}$ milliseconds. The second reaction is sending a fall alarm, which is triggered by fall detection. This is realized using a fall detection algorithm that requires sampling acceleration at regular intervals equal to $t_{\text {period }}$ milliseconds. The algorithm distinguishes two stages in fall detection: impact detection, with impact detected by acceleration exceeding a threshold value; and posture evaluation (see [13], [14] for a detailed description of the algorithm). Posture evaluation is performed $t_{l a g}$ milliseconds after an impact has been detected, and is used to establish if the person is lying down, in which case a fall has been detected. The acceleration is sampled with the same periodicity both for impact detection and posture evaluation. Hence the following timing requirements can be given for the second reaction: the acceleration sampling period $t_{\text {period; }}$ the lag between impact detection and posture evaluation $t_{l a g}$; and the maximum period of time between fall detection and sending an alarm $t_{\text {alarm }}$.

Both an assistance alarm and a fall alarm are sent using a radio transceiver and are received by external infrastructure which is outside the scope of the system. Therefore, the communication protocol (with its timing requirements) has to be part of the extended specification. 


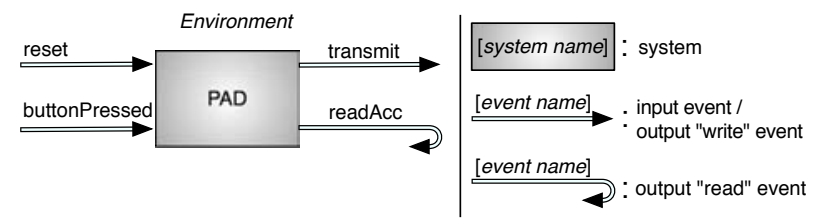

Figure 9. System-level model for personal alarm device. Input events: reset and buttonPressed. Output "read" event: readAcc (reading acceleration). Output "write" event: transmit (sending a fall alarm or an assistance alarm).

Non-functional requirements for the system include a relatively small size (since the system has to be worn on the body, for example, at the hip), and a low power consumption (as the device is to be powered by a battery).

\section{(2) Formulation of System-Level Model}

Analyzing system specification, we can distinguish two events that the system should react to: an assistance call realized as an interrupt from a button; and the person's fall. The interrupt from a button can be modeled as an external input event. The person's fall, however, is something that is detected by the fall detection algorithm which is internal to the system and hence it is not an external event. However, we can encode a periodic sampling of acceleration by the system as a reaction to a reset (an external input event) that starts up the system and triggers a reaction that includes sampling the acceleration (an external output "read" event) and posting a message with a delayed baseline that invokes another sampling after $t_{\text {period }}$ milliseconds, and so forth.

The timing requirements on the first reaction consist of a relative deadline $t_{\text {alarm }}$ milliseconds; the timing requirements on the second reaction are defined for each sampling that has a baseline equal to the baseline of the previous sampling plus $t_{\text {period }}$ milliseconds.

Note that while the hardware for the button, the accelerometer, and the radio transceiver are clearly a part of the system, the receiver of the alarm transmission is outside the developer's remit and should be viewed as an external service, not a system component. Thus the interface between the system and its environment is comprised on one hand, by reset interrupts and call button interrupts, and on the other hand, by the radio protocol used for communicating the alarms alongside the codes used to distinguish an assistance alarm from a fall alarm (see Fig. 9).

\section{(3a) Partitioning into Components}

Let us now consider partitioning into components of our device. Analyzing the specification and the systemlevel model (Fig. 9) we can see that the application will need the following independent resources: an acceleration sensor, a message sender (containing a radio transceiver), and a push button. Their independence warrants creating three separate components, each of them including both hardware and software parts (Fig. 10).

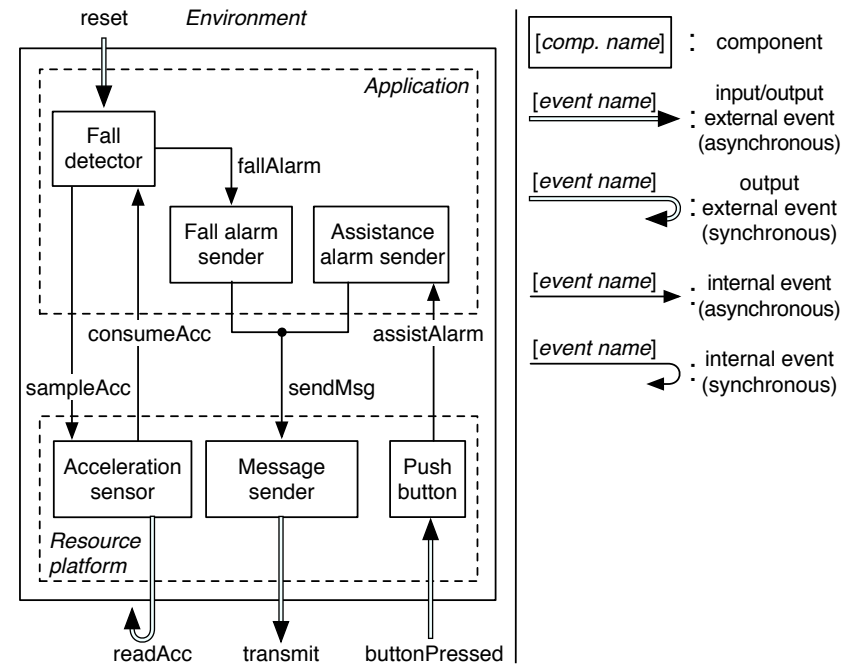

Figure 10. Component-level model for personal alarm device, with separation into a resource platform and a software application.

The next step is to define the interface of these components, bearing in mind that it should be complete but at the same time sufficiently abstract to accommodate various component implementations, which may possibly use different hardware to support the same functionality. The interface to the acceleration sensor should contain an input that can trigger sampling (sampleAcc), and an output that delivers the acceleration value once it has been acquired (consumeAcc). Note that to preserve reactivity and component independence, we cannot allow the caller to block waiting for the sampling to complete. It is therefore necessary to implement callback functionality in the acceleration sensor to specify to which component the measured acceleration should be delivered. This can be done either when the acceleration sensor is instantiated (a static callback), or by passing a pointer to a function each time sampling is triggered (a dynamic callback). Similarly, to achieve the desired level of generality, the interface of the message sender should only contain one input - sending a message (sendMsg), and one output - delivery of a received message, but the latter is superfluous for our application. Note that the message sender represents a clear example of a shared resource - it can be used by any of the independent tasks of (a) fall detection, and (b) handling an assistance call. As such, it will have to include either message queuing or some kind of arbitration to synchronize access to the resource transparently to the components that may want to use it simultaneously. The interface of the last resource component - the button - is very simple, as it only needs one output to deliver the button event and the target component can easily be set statically. These three components naturally form a platform with clearly defined functionality and interface between it and any possible application.

It now remains to partition the rest of the system the application - into components. Here two independent activities can be identified: fall detection and assistance 
call handling, resulting in two separate components. At the same time, it is appropriate to de-couple the fall detection algorithm from how the system should react to a detected fall. For our application, this involves creating a message and forwarding it to the message sender, which can be done by a separate component - a fall alarm sender. If assistance call detection in the application is similarly de-coupled from the reaction to it, we will have two very similar components - a fall alarm sender and an assistance call sender. A possible implementation is to create them as two instances of the same component, a general alarm sender, with some parameter set to different values at initialization. Alternatively, they can be viewed as two different components.

Timing requirements can be part of component specification as time constraints on the reactions. In this case, however, we skip this step and define the timing requirements directly at the object level.

\section{(3b) Search for Ready-Made Components}

In our example, the personal alarm device is developed from scratch and there are no components that can be reused in the design. However, let us consider what components could be used in the future in similar applications.

The first candidate for future use is, of course, the platform, consisting of an acceleration sensor, a message sender, and a push button (all components combining hardware and software). This is most natural because a platform is always defined as a collection of hardware and software resources that can be used by a range of possible applications. At the same time, it is not inconceivable that such components as an acceleration sensor, a message sender, or an alarm sender can be used separately in other designs.

\section{(3c) Hierarchical refinement of Component Structure}

In the case of the example system, there is no room for hierarchical refinement of component structure due to the system's simplicity.

\section{(4) Realization Using Reactive Objects}

The object-level model of the example system is presented in Fig. 11. The hardware parts have been identified and are shaded in the figure (their interfaces have been significantly simplified for presentation purposes).

It is clear that all resource components in our example require a mixed hardware/software implementation. In the acceleration sensor, the A/D controller object is used to abstract from the specific hardware interface of the A/D converter and to perform deserialization ${ }^{4}$. In the message sender, several objects are used to implement the network protocol, and a transparent sharing of the message sender between multiple components is provided by queuing

\footnotetext{
${ }^{4}$ Deserialization is required since $\mathrm{A} / \mathrm{D}$ conversion can only be performed on one channel at a time, but values from all three channels are sent to the application for analysis.
}
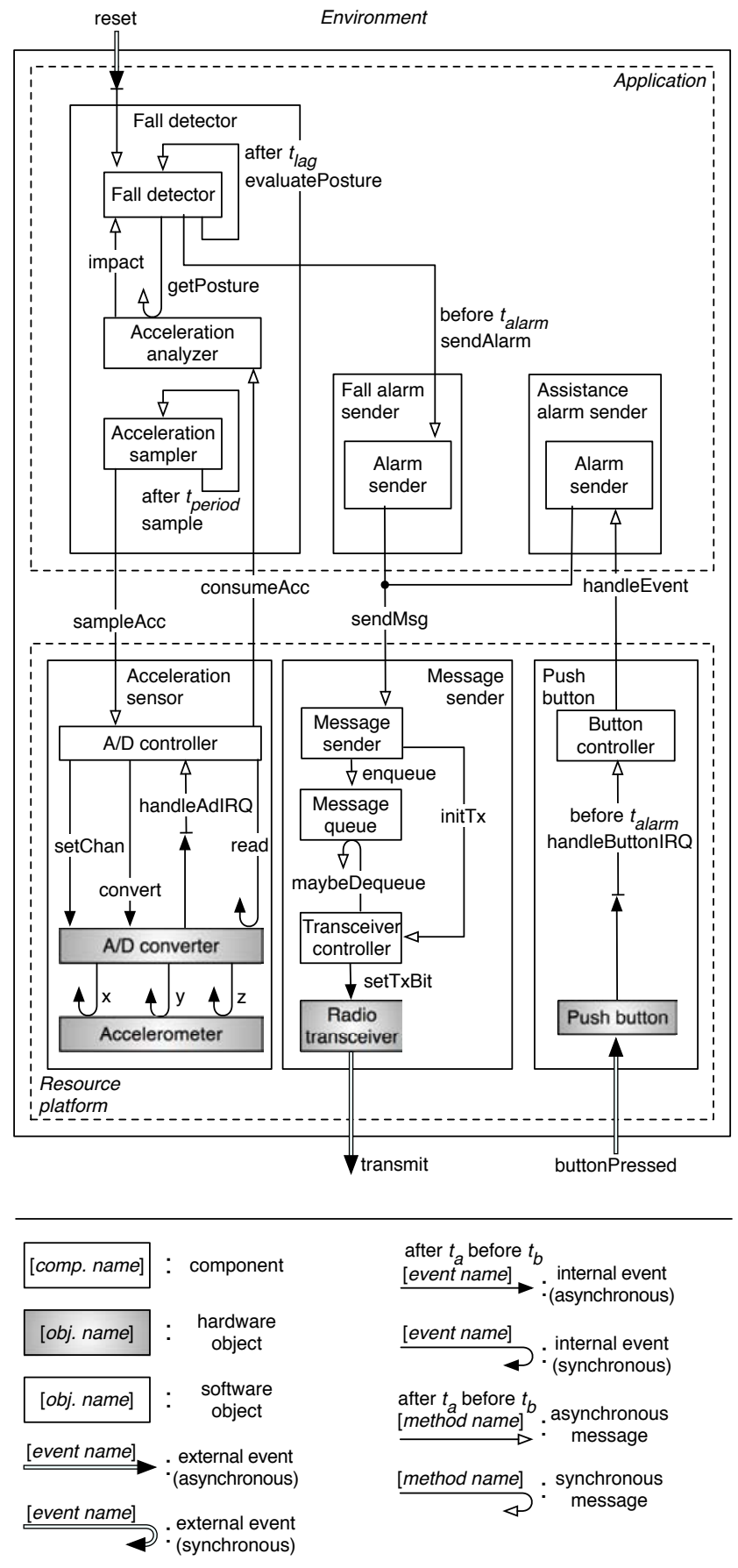

Figure 11. Object-level model for personal alarm device. Note the absence of the output "read" event readAcc, which has been redefined as three internal events $x, y, z$ reading different channels of an analog accelerometer.

incoming messages before sending. In the push button, a button controller functions as a simple interrupt handler.

The only purely software component that consists of more than one reactive object is the fall detector. The acceleration sampler object triggers sampling by posting an asynchronous message to the sampleAcc method of the acceleration sensor component. Sampling at predetermined intervals is achieved by the acceleration sample posting an asynchronous message to its own method sample with baseline delayed by $t_{\text {period }}$ : newBaseline $=$ 
currentBaseline $+t_{\text {period }}$.

The acceleration analyzer and fall detector objects cooperate to detect a fall. The acceleration analyzer posts an asynchronous message to the fall detector on detection of impact upon which the fall detector updates its internal state and posts an asynchronous message to its own method evaluatePosture delayed by $t_{\text {lag }}$. Once the evaluatePosture method is invoked, the person's posture is requested from the acceleration analyzer and if he or she is lying down, the fall detector posts an asynchronous message to the fall alarm sender specifying $t_{\text {alarm }}$ as deadline.

Assistance alarms are handled in a similar way. The application's handleEvent method is directly linked to the sendAlarm method in the assistance alarm sender. This method inherits the permissible execution window defined for the handleButtonIRQ method in the push button component.

\section{(5) Implementation in Timber}

The software part of the system was implemented in the programming language Timber (see section IV). As expected, the system structure presented in the model was complete and did not require any modifications; each reactive object in the model was implemented as such in Timber. Thus parallelism between system reactions was expressed at the object level in the model and preserved in the implementation. The implementation stage also involved writing Timber code for each method. All algorithms and functions were implemented; for example, a buffer holding sampled acceleration values was defined as a state variable in one of the objects, and a function was defined for filtering accelerometer data to remove noise in the signal.

The hardware platform defined in the model has not been implemented as yet. However, the software can be executed on any hardware platform that matches the presented model of the system, and we have verified that there exist COTS hardware parts that correspond to each reactive object in the model that should be implemented in hardware (acceleration sensor, radio transceiver, etc.).

\section{(6) Verification}

The Timber implementation of the system software was verified, partly by typechecking performed by the Timber compiler, and partly by simulation of the software in a Simulink-based Timber simulator. In the simulated environment, the functionality and the timing specification (preserved in the Timber code of the implementation) were tested by feeding the software simulation with real sensor data of recorded falls of several human subjects as well as their normal daily activity [14].

When software is executed in a simulated environment and not on a real hardware platform, no meaningful execution times are available. However, since the timing specification is preserved in the implementation in form of permissible execution windows for each reaction, it is possible in the simulation to choose any point within this window, which is correct in the sense that it corresponds to the timing behavior as expressed in the model. A natural choice is to let each reaction to execute (with zero execution time) at its baseline; this approach was used in our simulations. Note that verification of whether the worst-case execution times on a particular hardware platform allow the system to always meet the required deadlines is a separate issue and has not been part of the verification performed so far.

The verification of the software implementation demonstrated the validity of both the algorithm and its implementation, as the falls of the subjects were accurately detected in the simulation.

A detailed account of implementation and verification of the system will be published elsewhere.

\section{RELATED WORK}

The modeling and implementation approach realized in the Timber language can be compared to other solutions such as real-time synchronous languages (Esterel, SCADE, Lustre, etc. [15]) and time-triggered languages such as Giotto [16]. However, they are substantially different even if they can be seen as addressing the same design problems. For example, in synchronous languages, concurrency in system behavior is eliminated in the course of implementation, leading to a further separation between specification and model on one hand and implementation on the other hand. In Giotto, software is defined in terms of periodically executed tasks, reading inputs and writing outputs at pre-determined times, which is not particularly suitable for many embedded systems that exhibit a clearly reactive behavior and is not applicable to modeling hardware.

Our design approach is also different from that developed in the Ptolemy project [17]. The Ptolemy approach is a framework for assembly of concurrent components particularly suitable for modeling distributed systems. Essential to it is the notion of an actor, embodying the concept of active objects (as opposed to reactive objects). It can also be argued that the Ptolemy approach does little to bridge the gap between models and implementation, which is achieved in Timber by using executable models.

Component-based approach to design of (not necessarily embedded) real-time systems has been promoted by various extensions of real-time UML profile [18], with a number of tools already on the market (the most well-known probably are Rhapsody [19], ARTISAN Studio [20], and Rose-RT [21]). However, these solutions do not feature a true integration of timing requirements into functional specification, and do not completely solve the problem of modeling of mixed hardware/software systems.

Another design approach specifically targeting embedded systems is platform-based design [22]. This approach cannot really be considered component-based, as it concentrates on the methodology for separate development of a hardware platform, a system platform comprised by a 
hardware platform and hardware-software middleware (an API platform), and a software application that is developed for a given system platform. This can be contrasted with our approach where both a resource platform and a software application can be composed of multiple components, and where a resource platform can be designed together with an application; even if a platform is given, a model of the whole system is used to develop the software application. Our definition of a resource platform includes not only hardware components, but also software and mixed hardware/software components that can be utilized as resources by a range of applications.

\section{CONCLUSION}

The presented modeling framework allows for a unified, consistent modeling of both hardware and software. Integration of these models is beneficial for development of embedded systems as they often exhibit a great degree of interdependency between hardware and software, and the specification often describes the system as a whole rather than only its software part. At the same time, inclusion of timing requirements in a functional specification in the form of time-constrained reactions allows us to specify, reason about, and verify real-time properties of embedded systems. Moreover, our modeling framework enables the developer to offer platformindependent correctness/quality of service guarantees for hard/soft real-time systems, provided that the software can be scheduled on a given hardware platform so that all reaction deadlines are met.

By combining this modeling framework with component-based design techniques and by expressing system functionality using reactive objects, our approach draws from the strengths of component-based design as well as from event-based, reactive, concurrent, objectoriented programming models. It facilitates software re-use and maintenance as well as separate development of parts of the system. This approach is realized in the concrete software design methodology presented above.

Apart from addressing the issues of software complexity, interdependency between software and hardware, and complying with the timing requirements, our approach also allows to clearly define the notion of a resource platform as a combination of hardware and software resources. A resource platform can be designed to serve as a base for a whole range of related applications, decreasing the overall development costs and time to market.

The presented software design methodology can be used both in the case when software is developed alongside a hardware platform (the latter being assembled from existing hardware parts) and in the case when such a platform is given from the start. In both cases, a platform is instantiated using some implementations of hardware and/or software components depending on performance, power consumption, and other non-functional requirements.
The design approach presented in this article requires further development in the following directions: formalization of component structure; creating design tools supporting the methodology; and investigating the role of other requirements, such as power consumption, in the design process. The power consumption issue is especially challenging since it introduces new constraints and modeling parameters to deal with. However, with today's rapid increase of battery-powered embedded systems, this is a very important issue to address. A holistic view of both timing and and power consumption will offer new and interesting possibilities in the area of embedded system design.

\section{ACKNOWLEDGMENT}

This work was supported in part by the Knowledge Foundation in Sweden under a research grant for the project SAVE-IT, the EU SOCRADES project, and the EU Interreg III A North Programme grant 304-137232005.

\section{REFERENCES}

[1] B. Bouyssounouse and J. Sifakis, Embedded Systems Design: The ARTIST Roadmap for Research and Development, ser. Lecture Notes in Computer Science. Berlin, Germany: Springer-Verlag, 2005.

[2] C. Atkinson, C. Bunse, H.-G. Gross, and C. Peper, Component-Based Software Development for Embedded Systems: An Overview of Current Research Trends, ser. Lecture Notes in Computer Science. Berlin, Germany: Springer-Verlag, 2005.

[3] J. Nordlander, M. P. Jones, M. Carlsson, and J. Jonsson. (2005) Programming with time-constrained reactions. [Online]. Available: http://pure.ltu.se/ws/fbspretrieve/441200

[4] J. Nordlander, M. P. Jones, M. Carlsson, R. B. Kieburtz, and A. Black, "Reactive objects," in Fifth IEEE International Symp. on Object-Oriented Real-Time Distributed Computing, 2002, pp. 155-158.

[5] W. Yi, P. Pettersson, and M. Daniels, "Automatic verification of real-time communicating systems by constraintsolving," in Proc. of the 7th International Conference on Formal Description Techniques, 1994, pp. 223-238.

[6] K. G. Larsen, P. Pettersson, and W. Yi, "Model-Checking for Real-Time Systems," in Proc. of Fundamentals of Computation Theory, ser. Lecture Notes in Computer Science, no. 965,1995 , pp. $62-88$.

[7] J. Bengtsson, K. Larsen, F. Larsson, P. Pettersson, and W. Yi, "Uppaal-a tool suite for automatic verification of real-time systems," in Proc. of the DIMACS/SYCON workshop on Hybrid systems III : verification and control. New York, USA: Springer-Verlag, 1996, pp. 232-243.

[8] T. Amnell, et al., "Uppaal - now, next, and future," in Proc. of the 4th Summer School on Modeling and Verification of Parallel Processes. London, UK: Springer-Verlag, 2001, pp. $99-124$.

[9] L. Sha, et al., "Real-time scheduling theory: A historical perspective," Real-Time Systems, 2004.

[10] P. Lindgren, J. Nordlander, L. Svensson, and J. Eriksson. (2005) Time for timber. [Online]. Available: http: //pure.ltu.se/ws/fbspretrieve/299960

[11] M. Carlsson, J. Nordlander, and D. Kieburtz, "The semantic layers of timber," in First Asian Symp. on Programming Languages and Systems: APLAS, ser. Lecture Notes in Computer Science, 2003, pp. 339-356. 
[12] The Timber language homepage. [Online]. Available: http://www.timber-lang.org

[13] M. Kangas, J. Wiklander, I. Vikman, L. Nyberg, P. Lindgren, and T. Jämsä, "Sensorband fall detector prototype: Validation through data collection and analysis," in The 2:nd Int. Symp. on Medical Information and Communication Technology (ISMICT'07), 2007.

[14] M. Kangas, I. Vikman, J. Wiklander, P. Lindgren, L. Nyberg, and T. Jämsä, "Sensitivity and specificity of fall detection in people aged 40 years and over," Gait \& Posture, 2009, In press.

[15] A. Benveniste and G. Berry, "The synchronous approach to reactive and real-time systems," Proc. IEEE, vol. 79, no. 9, pp. 1270-1282, 1991.

[16] T. Henzinger, B. Horowitz, and C. Kirsch, "Giotto: A timetriggered language for embedded programming," Proc. IEEE, vol. 91, no. 1, pp. 84-99, 2003.

[17] Y. Zhao, J. Liu, and E. Lee, "A programming model for time-synchronized distributed real-time systems," 13th IEEE Real-Time and Embedded Technology and Applications Symposium: RTAS, pp. 259-268, 2007.

[18] S. Graf, I. Ober, and I. Ober, "A real-time profile for UML," Int. J. on Software Tools for Technology Transfer, vol. 8, no. 2, pp. 113-127, 2006.

[19] E. Gery, D. Harel, and E. Palatshy, "Rhapsody: A complete life-cycle model-based development system," in The Third Int. Conf. on Integrated Formal Methods, 2002, pp. 1-10.

[20] Artisan Software Tools. [Online]. Available: http://www. artisansw.com/

[21] Rational Rose Technical Developer. [Online]. Available: http://www.ibm.com/software/rational

[22] A. Sangiovanni-Vincentelli, "Defining platform-based design," EEDesign of EETimes, 2002.

Jimmie Wiklander is a Ph.D. student in Computer Science at Luleå University of Technology. He is also a student with SAVEIT industrial graduate school, which focuses on real-time and safety-critical systems. His research area is component-based design of embedded real-time systems.

Jens Eliasson is an assistant professor in Computer Science at Luleå University of Technology and holds a Ph.D. in Industrial Electronics from the same university. His main research areas are low-power design of Embedded Internet Systems (EIS) and wireless sensor networks.

Andrey Kruglyak is a Ph.D. student in Computer Science at Luleå University of Technology, Sweden. His research interests include component-based design of real-time systems, objectoriented programming and programming language design.

Per Lindgren holds the position of chaired professor in Embedded Systems at Luleå University of Technology. He is currently heading a group of Ph.D. students in the area of embedded system design, with a focus on real-time, low-power software and hardware architectures for Embedded Internet Systems. He is a member of the ARTES++ reference group, and member of the ARTEMIS Architecture group, having contributed to the ARTEMIS Strategic Research Agenda.
Johan Nordlander is an assistant professor in Computer Science at Luleå University of Technology and holds a Ph.D. in Computing Science from Chalmers University of Technology. His research interests focus on programming language design and semantics, compilers, real-time systems, and practically applied program analysis and verification. 